\title{
LA PSIQUIATRÍA FENOMENOLÓGICA DE LA ESQUIZOFRENIA SEGÚN THOMAS FUCHS
}

\author{
Rosa María de Barrio Rodríguez \\ IES Alpujarra, Órgiva, Granada
}

\begin{abstract}
Resumen: Esta investigación trata sobre la esquizofrenia como trastorno de la intercorporalidad. Ésta según Thomas Fuchs, sucesor de Karl Jaspers en la cátedra, y como él, psiquiatra y filósofo, no es un trastorno ni primariamente cognitivo ni neurológico, sino de la intersubjetividad encarnada. Al explorar esta tesis, desarrollaremos gran parte de los conceptos de la antropología filosófica elaborada por Fuchs: resonancia corpórea, transparencia corpórea, intercorporalidad e interafectividad.

Palabras clave: Thomas Fuchs; Psiquiatría fenomenológica; Esquizofrenia; Intercorporalidad; Descorporalización; Intersubjetividad encarnada; Esquemas corporales; Delirios;

THE PHENOMENOLOGICAL PSYCHIATRY OF SCHIZOPHRENIA ACCORDING TO THOMAS FUCHS
\end{abstract}

Abstract: This paper focusses on schizophrenia as an intercorporeal disorder. This, according to Thomas Fuchs, successor of Karl Jaspers in the chair, and like him, psychiatrist and philosopher, is not primarily a cognitive or neurological disorder, but of incarnate intersubjectivity. In exploring this thesis, we will develop a large part of the concepts of the philosophical anthropology elaborated by Fuchs: corporeal resonance, corporeal transparency, intercorporality and interafectivity. Keywords: Thomas Fuchs; Phenomenological psychiatry; Schizophrenia; Intercorporality; Disembodiment; Incarnate intersubjectivity; Bodily schemes; Delusions;

Recibido: 27 de abril de 2021

Aceptado: 12 de mayo de 2021

DOI 10.24310/NATyLIB.2021.vi15.12434 
A la memoria de mi padre. Mi Quijote.

\section{I.1. Thomas Fuchs y la psiquiatría fenomenológica}

Thomas Fuchs es un conocido psiquiatra, filósofo y profesor de la Universidad de Heidelberg. Su trabajo se enfoca en la rehabilitación de la psiquiatría fenomenológica. Ha realizado importantes y reconocidas aportaciones a la fenomenología, la neurociencia cognitiva y la psicopatología. Más concretamente, su investigación se centra en la intersubjetividad, la corporalización —embodiment - y la temporalidad.

Thomas Fuchs, sucesor de Jaspers en la cátedra, y como él, psiquiatra y filósofo, prosigue sus pasos realizando una psiquiatría fenomenológica que tiene en cuenta el rico bagaje de la fenomenología de Husserl, Heidegger, Merleau-Ponty o la filosofía fenomenológica de la mente de Gallagher entre otros. Como fenomenólogo está muy interesado en la descripción de nuestras experiencias o vivencias; como psiquiatra, su esmerada descripción y comprensión de las vivencias patológicas nos permiten entender el mundo y la conciencia de pacientes mentalmente afectados. En esto sigue la línea abierta por Jaspers en su Psicopatología general, cuando insistía en el valor del qué vivimos frente al por qué habitual en las ciencias naturales, la medicina incluida, que con su ansia de explicar atiende exclusivamente a los síntomas y busca las alteraciones neurobiológicas, olvidando el sentido de las experiencias, su modo de presentación y el mundo vivido por esos sujetos.

\section{I.2. Fuentes de esta investigación: Merleau-Ponty y Minkowski}

La fenomenología de Fuchs se sitúa y toma inspiración en la fenomenología del cuerpo vivido (Leib) frente al cuerpo objetivado (Körper), que se desarrolló en el siglo XX, y cuyos principales exponentes son Husserl y Merleau-Ponty. Fuchs se propone explícitamente desarrollar el concepto de intercorporalidad - intercorporéité - que Merleau-Ponty (1960) apunta en un 
breve texto incluido en Signes, con el que intenta complementar la fenomenología de la intersubjetividad husserliana.

Este análisis puede considerarse como una articulación del concepto merleau-pontiano de "intercorporalidad" (intercorporéité) con el que trata de complementar la explicación husserliana de la intersubjetividad como la constitución o "apresentación" del otro por un ego consciente. La intercorporalidad significa un entrelazamiento prerreflexivo de cuerpos vividos y vivientes, en el que mi propio cuerpo es afectado por el del otro tanto como el del otro por el mío, conduciendo a una comunicación encarnada (Fuchs, 2016: 200).

La otra fuente de la fenomenología psiquiátrica que Fuchs realiza sobre la esquizofrenia es Minkowski. En su clásica monografía La schizophrenie de 1927 Minkowski caracterizó esta enfermedad mental, siguiendo a Bergson, como una pérdida de contacto vital con la realidad. A diferencia de las privaciones sensoriales con algunos aspectos de la realidad, como la ceguera o la sordera, la esquizofrenia no es un trastorno fundamentalmente cognitivo, sino vital. Es un trastorno que no afecta tanto a la cognición ni a la reflexión, pese a los delirios y alucinaciones, como al yo y a la vida. A diferencia de enfermedades como la depresión o los trastornos maniáticos, en los que hay un fuerte contacto con la realidad, el esquizofrénico pierde la cercanía vital de lo real, el ser afectado por lo real de modo que éste signifique algo para él, ya sea interesándolo, preocupándolo, aterrorizándolo, etc. Esto da lugar a la mayor separación posible entre el yo y el intelecto, o entre la vida y la reflexión: el yo reflexivo, observador, se divorcia del sí mismo corporal: esto es el yo que se descorporeiza.

\section{I.3. Tema y tesis de esta investigación}

Esta investigación trata sobre la esquizofrenia como un trastorno de la intersubjetividad encarnada, o lo que es lo mismo de la intercorporalidad. La descripción filosófica de la esquizofrenia es especialmente importante porque 
este trastorno mental desafía aquello que Inmanuel Kant consideró la esencia de la autoconciencia: el que un sujeto pueda identificar como «suyas» representaciones, acciones, percepciones, o incluso su cuerpo (Fuchs, Röhricht,2017:4). Que mis sensaciones, sentimientos, afectos, movimientos y mi cuerpo sean «míos» significa que los vinculo conmigo, que no me encuentro respecto de ellos en una situación de lejanía. Esta cercanía, por la cual digo que son míos, es al yo, a la vida, y no al intelecto reflexivo. Éste yo o esta vida no es el sujeto trascendental kantiano, sino

$[\ldots]$ la dación en primera persona de toda experiencia [...] que incluso queda preservada cuando se pierde la memoria autobiográfica, como en la amnesia o en la demencia, o cuando se deteriora el sentido a largo plazo del futuro (Fuchs, Röhricht, 2017:5).

Incluso en estas graves patologías el paciente «sabe» inmediatamente, sin reflexión ni introspección, que es, que tiene cuerpo, sensaciones, sentimientos, afectos, movimientos, cuerpo, y que puede actuar en el mundo y con otras subjetividades encarnadas, que también saben de sí en los mismos términos. Pues bien, en la esquizofrenia el paciente característicamente acusa angustia o terror ante lo que considera la pérdida de ese sí mismo encarnado del que sabe inmediata y prerreflexivamente. Se produce una debilitación de su conciencia vital más o menos grave, que puede llegar a alcanzar la sensación de una pérdida total de su mismidad psicosomática pasada.

Como recuerda Fuchs, esta conciencia vital, ha sido denominada ipseidad por algunos fenomenólogos como Sartre (1943), Henry (2000) o Zahavi (2005). Fuchs (Fuchs, Röhricht, 2017) prefiere hablar de «yo básico». Al yo que comparece en la conciencia reflexiva, lo denomina yo extendido, personal o reflexivo.

Además, la psicopatología fenomenológica es necesaria para comprender qué están viviendo esos pacientes y cómo lo viven. Estas preguntas no son respondidas por la simple enumeración externa y objetiva de un conjunto de síntomas, mucho menos por la investigación neurológica.

Fuchs y Röhricht (2017) han compendiado los resultados de las investigaciones sobre la autoconciencia que del cuerpo tienen los pacientes esquizofrénicos en la siguiente lista de síntomas: 
Sensaciones corporales anormales (cenestesias), cualitativamente desacostumbradas.

Desomatización/despersonalización correspondiente a pérdidas de límites, vividas subjetivamente como miedo a la desintegración corporal, y que dan lugar a esfuerzos de reconstrucción del comportamiento (ej. exposición al espejo, rituales compulsivos).

Pérdida de control sobre el propio cuerpo (esto es, fenómenos de pasividad somática).

Centralización del esquema corporal: percepción distorsionada de las partes periféricas del cuerpo (estimación a la baja de las extremidades inferiores conectado con el sentimiento de que el cuerpo es inusualmente pequeño.

Movimientos estereotipados con contacto consigo repetitivo, autoestimulación a través de palmadas o golpeando partes del cuerpo contra objetos (Fuchs, Röhricht, 2017: 5).

Thomas Fuchs (2005), y en general filósofos y psiquiatras - como Gallagher (2004) o Saas y Parnas (2003) - que adoptan un punto de vista fenomenológico, se sitúan como alternativa teórica a entender la esquizofrenia bien como un trastorno o fundamentalmente cognitivo, o meramente neurológico. Según Sanneke de Haan y Thomas Fuchs:

[...] entre [estos enfoques] se encuentra la perspectiva fenomenológica, cuyos proponentes argumentan que podemos entender mejor la esquizofrenia como un trastorno de sí mismo encarnado. El sentido prerreflexivo básico del sí mismo está debilitado, lo que a su vez se muestra en déficits en el nivel neurológico que influyen en el funcionamiento cognitivo superior (Sanneke, Fuchs, 2010: 328).

Este trabajo de investigación tiene como cometido explorar esta tesis de Fuchs sobre la esquizofrenia, es decir tener en cuenta la relevancia del papel encarnado de la subjetividad en la psicopatología de la esquizofrenia. ${ }^{1}$

\footnotetext{
${ }^{1}$ Fuchs tiene una visión de la esquizofrenia más compleja, pues a esta tesis sobre la descorporalizacion hay que sumar que considera a «la esquizofrenia como un trastorno de la autoconciencia de base (ipseity) (Fuchs, Röhricht, 2017: 5). Los límites de esta investigación
} 
Esta tesis se presenta como alternativa a dos hipótesis que consideran la esquizofrenia desde los dos polos del dualismo cartesiano: entenderla como una enfermedad puramente somática, o puramente cognitiva. Entender los trastornos de esquizofrenia como trastornos del sí mismo encarnado es un intento de recuperar la unidad de la persona.

Este primer epígrafe de este artículo tiene como cometido exponer la visión que Fuchs tiene sobre la intercorporalidad. Esta exposición se lleva a cabo en el siguiente supepígrafe. Estos análisis constituirán la base de la psiquiatría fenomenológica que Fuchs realiza sobre las vivencias de la corporalidad de los pacientes esquizofrénicos, tarea del segundo supepígrafe de esta primera parte de mi investigación. Además de explorar la fecundidad de este concepto, Fuchs, junto con algunos destacados fenomenólogos contemporáneos - Gallagher (2001: 83-108), De Jaegher (2009: 465-486) o Froese (2012: 205236) - , han forjado y desarrollado la noción paralela de interafectividad.

\section{La intersubjetividad encarnada}

Nuestro cuerpo nos inserta en el mundo, y nos pone en relación inmediata con los demás. Pero lo hace de un modo que podríamos denominar silencioso, no comparece en nuestra conciencia su funcionamiento para percibir, actuar, etc., A esta propiedad de la corporeidad en relación con la conciencia Fuchs (2001: 323-326) la denomina dimensión tácita, es decir callada, silenciosa, que debe ser inferida, que se sobreentiende pero que no suele comparecer explícitamente. Por eso, uno de los fundadores de la antropología filosófica, Helmuth Plessner solía mantener que la inmediatez de nuestro estar inmediatamente en el mundo, es mediada por el cuerpo, sin cuya continua actividad tácita no estaríamos en el mundo de objetos con los que hacemos nuestra vida, ni ante otras personas. El cuerpo, pues, media tácitamente el mundo.

Esta dimensión tácita del cuerpo se logra mediante la adquisición de hábitos. Con ellos tomamos posesión de las diferentes dimensiones de nuestra corporeidad. En esta adquisición nuestra conciencia y nuestra atención juega un papel crucial. Pero una vez adquirida la técnica corporal, generalmente

obligan a tratar de esta segunda tesis instrumentalmente, y no de manera directa. Lo haré al tratar del cuerpo vivido y de su conciencia prerreflexiva, que Fuchs distingue de la autoconciencia reflexiva. 
aprendida intersubjetivamente, la conciencia se retira, y podemos llevar a cabo los movimientos y actividades corporales requeridas para esa acción cuasi automáticamente, sin prestar atención explícita a los elementos coordinados en el esquema corporal que hemos adquirido. En lo que sigue voy a tratar en primer lugar de esta dimensión tácita y su relación con la intersubjetividad. Después hablaré de cómo esta dimensión aparece clamorosamente, es decir deja de ser tácita, en las vivencias, experiencias y sentimientos del esquizofrénico.

\section{II.1. Interacción e intersubjetividad encarnada}

Según Fuchs, la interacción humana está, desde la más tierna infancia, mediada corporalmente. El cuerpo nos integra en el mundo circundante, y actúa como mediador en dicho mundo. Pero la perspectiva de Fuchs no es la de un sujeto aislado, sino la de sujetos encarnados que entran directa e inmediatamente en contacto, en interacción. Sujetos que son afectados en su cuerpo, vivo y vivido, por el cuerpo vivido y viviente de otro sujeto - por su mirada, su expresión facial, sus gestos y movimientos-. Este ser afectado crea un acoplamiento, emparejamiento, entrelazamiento o coordinación corporal inconsciente entre las percepciones y las acciones de esas subjetividades. Por eso, desde su punto de vista, los sujetos que entran en relación no están, al modo cartesiano, encerrados en la coraza de su conciencia, y así separados radicalmente del otro. Como ha puesto de relieve Pedrosa:

Según Fuchs la fenomenología tiene la virtud de no considerar separados a los sujetos entre sí y acceder a estos desde su interioridad, como si nos sumergiéramos en ellos, o estuviéramos sumidos en ellos (Pedrosa, 2018: 13).

La descripción fenomenológica, que atiende al cuerpo vivido — Leib - , se distingue de la que hacen las ciencias naturales, que consideran al cuerpo como un objeto exterior entre otros - Körper-, en que accede directa e inmediatamente a la interioridad subjetiva. Para las ciencias empíricas la subjetividad no trasparece necesariamente en el cuerpo. Éste es el implícito del dualismo o separación radical entre cuerpo y mente como dos sustancias distintas. Se trata del viejo problema, ya registrado por Descartes, sobre la 
posibilidad de engañar y fingir. El cuerpo, en las relaciones intersubjetivas $j^{\text {es }}$ un medio opaco, o puede cobrar algo así como una «trasparencia intersubjetiva» (Fuchs, 2005:103)? Si los cuerpos de los sujetos que interactúan son capaces de este tipo de transparencia, ¿en qué condiciones la logran?

Es cierto que las interacciones entre dos sujetos encarnados son, cuando tienen lugar de modo inmediato y directo, corporales. Los sujetos interactúan a través de sus miradas, sus gestos expresivos, los tonos de su voz, los movimientos del resto de su cuerpo y, en ocasiones, su contacto físico. Al concentrar la atención fenomenológica en las interacciones del sujeto con los entes intramundanos o con otros sujetos, Fuchs elabora

[...] una teoría sobre la vivencia del propio cuerpo en las interacciones. Los tipos posibles de interacciones sólo pueden ser de dos tipos: unidireccionales y mutuas. O interactúo con un objeto inanimado o interactúo con otro sujeto vivo. En sendas interacciones vivimos nuestro cuerpo. $\mathrm{Y}$ en la segunda nuestro cuerpo también es vivido por otro (Fuchs, 2005: 15).

\section{II.2. Resonancia corpórea y trasparencia intersubjetiva}

Fuchs se está pues situando no en la perspectiva de la autoconciencia reflexiva, sino en la del viviente, que vive su propio cuerpo, el de los otros entes que lo rodean y con los que trata, y el de los otros sujetos que establecen con él conductas mediadas por su propia corporeidad.

Además, esta vivencia del cuerpo de los otros sujetos vivos es también tenida por éstos respecto del suyo. Así se produce el fenómeno que Fuchs denomina «resonancia corpórea»:

[...] el otro resuena en nuestro cuerpo, que se transforma así en el espacio y en medio en que resuena el otro. [...] Sentimos al otro en nuestro propio cuerpo (Fuchs, 2005: 16).

El otro, con su subjetividad, resuena a través de las conductas corporales con que interactúa con nosotros, en nuestra propia corporeidad. Ahí, en nuestro propio cuerpo, sentimos sus intenciones, sus pensamientos, la percepción que tiene de sí mismo, etc.

La resonancia corpórea tiene como su condición de posibilidad la interacción, las prácticas y actividades comunes, recíprocas, en la que el sujeto se 
entiende no como una conciencia ante la que se abre el mundo, y quizá otras conciencias, sino como un agente en el mundo desarrollando sus actividades entre otros agentes, con ellos y, a veces, contra otros agentes. Esta perspectiva, la del encuentro cara a cara, la de la interacción corporal inmediata, desvanece los problemas cartesianos sobre la autoconciencia del otro, y el consiguiente solipsismo. La psique del otro no está escondida tras su corporalidad, sino que resuena en mi cuerpo a través de sus conductas corporales. Porque sus intenciones, pensamientos y autoconciencia resuenan en mi corporeidad, no puedo considerarlo como un mero cuerpo físico con el que me encuentro.

Por otra parte, el fenómeno de la resonancia también es intercorporal. La fenomenología contemporánea ha analizado detenidamente co-experiencias o co-vivencias como la del reír juntos. Esta vivencia, por ejemplo, no es explicable como la mera adición de sujetos que ríen por separado. La alegría de uno resuena en el cuerpo del otro, le afecta, lo pone alegre. La alegría de uno no se da al margen, o independientemente de la alegría del otro. Así es como mi psiquismo es-con - mit-sein - otros psiquismos a través de un cuerpo que está siendo, viviendo y actuando con otros.

La fenomenología del reír juntos muestra además otro aspecto sumamente revelador. Los agentes que interaccionan corporalmente de forma directa se encuentran además sintonizados afectivamente. Vivo, por resonancia corporal, las emociones y los afectos que el otro expresa corporalmente. También vivo, en mi propio cuerpo, las intenciones que el otro despliega mediante su comportamiento corporal. Esta sintonía es prerreflexiva, previa a cualquier conceptualización o reflexión sobre sus móviles o fines, o a cualquier declaración lingüística sobre lo que hace, busca, o pretende. Se da cierto entendimiento, mejor sería decir coincidencia entre sus emociones, afectos e intenciones y los míos. Esta coincidencia es la sintonía - o acuerdo-emocional. No sólo las ideas de uno pueden coincidir con las de otro, también las emociones pueden coincidir, como es obvio, y lo hacen generalmente antes que las ideas.

\section{II.3. La estructura "como sí» de la intersubjetividad y la transferencia de los esquemas corporales}


Merleau-Ponty (1945) describió la resonancia corporal como la «transferencia del esquema corporal» de un sujeto encarnado a otro. Un esquema corporal es una forma - Gestalt - que adopta nuestro cuerpo, intencional y prerreflexivamente. Un modo de responder corporalmente a las solicitudes que le hacen los entes del mundo en que vive. Se trata de un patrón o disposición de un conjunto muy vasto de elementos corporales - tendones, músculos, articulaciones, y de la fuerza, dirección, presión, etcétera, con que los usamos - que nos permiten desde la vocalización y el caminar erecto hasta la interpretación de un instrumento musical o el uso de instrumentos de precisión. El esquema corporal es prerreflexivo. Si intentamos representar todos y cada uno de sus elementos conscientemente, el movimiento se vuelve torpe, vacilante, o simplemente, colapsa - apraxias-.

La intencionalidad del cuerpo no es la misma que la de nuestras representaciones mentales. La intencionalidad del cuerpo es lograda a través de hábitos que familiarizan nuestro cuerpo con las actividades y realizaciones que lleva a cabo, permitiendo el diálogo entre el cuerpo y los entes del mundo circundante, y entre el sujeto encarnado y otros sujetos. Los esquemas corporales de un sujeto son compartidos con otros sujetos con la misma inculturación o que desarrollan las mismas actividades con él. Forman así lo que podríamos denominar un «common sense» (Vico, Blankenburg), un conjunto de «técnicas corporales» (Mauss) aceptadas implícitamente por una comunidad como el modo natural, evidente, o normal estar en el mundo y entre los otros. Según Fuchs (2001), y esta es una aportación original al clásico tema del common sense, el sentido común de una colectividad está encarnado, ese conjunto de evidencias sobre lo natural, lo bueno, lo apropiado, transmitidas y compartidas culturalmente, variables por tanto entre diferentes colectividades, tiene una base que no es reflexiva, sino corporal. Precisamente por ello:

La comunicación o comprensión de los gestos se logra a través de la reciprocidad de mis intenciones y de los gestos de los otros, de mis gestos y las intenciones discernibles en la conducta de otras personas. Es como si las intenciones de la otra persona inhabitasen mi cuerpo y las mías el suyo (Merleau-Ponty,2005: 98).

Fuchs subraya en este texto de Merleau-Ponty la estructura «como sí» de la comunicación gestual. En las situaciones intercorporales, en acciones cooperativas, en la observación mutua y recíproca, las intenciones del otro con 
quien interactúo parecen como si inhabitasen en mí. Se logra así la trasparencia intersubjetiva de la que hablábamos anteriormente. Esta trasparencia recíproca de las intenciones y percepciones del otro se logra en la propia corporeidad. Evidentemente sus intenciones o sus percepciones no animan mi cuerpo, sólo es como si lo hiciesen. Por eso, según Fuchs, este inhabitar en mí el otro es virtual. En modo alguno es real o en acto. Entre agentes no afectados de patologías mentales como el autismo o la esquizofrenia, el agente siempre discierne sus intenciones de las de los demás. La autoconciencia mantiene su seipseigualdad. Pero comprendo los gestos del otro porque mi cuerpo puede esbozarlos para atender a determinados objetivos, para alcanzar tales o cuales fines. Por eso, Merleau-Ponty podía hablar de transferencia de esquemas corporales. No sólo percibo sus movimientos, sino que la intencionalidad de nuestro propio cuerpo nos descubre sus intenciones, los objetivos de su acción.

En la base de esta estructura «como sí» se encuentra el esquema corporal propio: la disposición intencional de nuestro cuerpo en relación con el mundo circundante. Así es «como» estamos, nos comportamos o actuamos ante tal o cual objeto. Nuestro esquema corporal actual es el modo «como» estamos actualmente en el mundo. Pero este esquema corporal podemos usarlo para diseñar futuras acciones. Entonces se virtualiza. Ante ese objeto, mi cuerpo tendrá que comportarse «como sí» ... También podemos usarlo, no para prever futuras acciones nuestras, sino para comprender acciones ajenas. $\mathrm{Su}$ cuerpo se dispone «como si» tuviese tal intención respecto de nosotros. Transfiero así mis esquemas corporales a otro sujeto.

Así, la simulación es el medio de comprender a los otros usando mi propio cuerpo como un espejo de sus intenciones y actitudes; en otras palabras, mediante la simulación el cuerpo obtiene trasparencia intersubjetiva (Fuchs, 2005: 103).

Se trata de una transferencia que realiza un sujeto a otro sobre el sentido de sus acciones corporales. Esta atribución de significado puede ser errónea, pero no está basada en una inferencia o una deducción mental, sino en la intencionalidad del cuerpo vivido. Éste, para alcanzar un determinado fin se dispone de tal o cual modo, ha adquirido tales destrezas o habilidades, y por eso depende de las capacidades que tiene el sujeto que realiza la transferencia para actuar en su mundo. Estas capacidades pueden verse mermadas, por 
ejemplo, en determinadas enfermedades mentales. Merma que se observa en la torpeza de movimientos, y la inhibición que presentan algunos enfermos con patologías como la esquizofrenia. Trataré las consecuencias de este fenómeno en el siguiente epígrafe dedicado a las patologías de la interacción encarnada.

\section{La esquizofrenia como trastorno de la intersubjetividad encarnada}

\section{III.1. La descorporalización del sí mismo en la esquizofrenia}

Una de las vivencias prototípicas de la esquizofrenia es la de la alienación. Quizá teniendo como analogado principal de la enfermedad mental a la esquizofrenia se ha hablado en general, desde tiempos de Philippe Pinel, de alienados para referirse a los enfermos mentales. La alienación, la experiencia de extrañamiento, es característica, con mayor o menor gravedad, de esta enfermedad. Los esquizofrénicos suelen sufrir un «extrañamiento de sí mismos, incluyendo a sus cuerpos, y respecto de otras personas en su mundo circundante» (Haan, Fuchs, 2010:329). La alienación es pues la vivencia de sentirse extraño a uno mismo, de sentir extraño el propio cuerpo, o determinadas dimensiones de él, de sentirse extraño en medio del mundo y de los demás. Los pacientes expresan que ya no saben quiénes son realmente, incluso dudan sin son incluso una persona.

Se trata de una escisión o una ruptura de la subjetividad y la conciencia respecto de otras dimensiones constitutivas de la propia realidad personal, que terminan disolviendo o desrealizando esas dimensiones o los vínculos que el esquizofrénico guarda con su mundo. Esta disolución también afecta, necesariamente, a la autoconciencia del paciente, puesto que ha perdido los puntos de referencia básicos de su identidad:

En la esquizofrenia, el "conocimiento" autoevidente de quién eres y de qué quieres, del mundo y de tu lugar en él, ya no está a tu disposición (Haan,Fuchs, 2010: $331)$.

Según Fuchs, en algunas enfermedades mentales, y manifiestamente en el autismo y la esquizofrenia la persona sufre, típicamente, una descorporalización o desencarnación del yo. Podríamos calificar a esta tesis, acerca de la 
vivencia del propio cuerpo por la persona aquejada de esquizofrenia, como fuerte. Según Fuchs:

[...] los análisis fenomenológicos sugieren que la persona esquizofrénica sufre de lo que podría denominarse una descorporalización del sí mismo — disembodiment of the self - Esa persona ya no inhabita su cuerpo, en el sentido de usar su estructura implícita, sus hábitos, sus realizaciones automáticas, como un medio, que se da por descontado, para relacionarse con el mundo (Fuchs,2005: 101).

El cuerpo del esquizofrénico ya no actúa como un medio que lo instale en el mundo, y le permita relacionarse con los entes que hay en él, o interactuar con otras personas. Parece como si sus esquemas corporales se disolviesen, es decir, de las formas — Gestalten - o patrones — patterns - que adopta el cuerpo intencionalmente. Así como hay un proceso de formación de hábitos y técnicas corporales, también cabe su disolución. Los hábitos son los modos en que la persona toma posesión de su organismo. No basta ser bípedo anatómicamente para andar. Es preciso «saber andar», es decir adquirir el conjunto de esquemas corporales que permiten un deambular grácil y coordinado. La persona, gracias a sus hábitos, tiene y puede con su cuerpo. Consiguientemente entonces, sólo gracias a los hábitos la persona puede decir que su cuerpo, y sus dimensiones, son suyas. Pues bien, en la esquizofrenia sucede, como indica Fuchs (2005), que «se debilita la "mioidad" — mineness - tácita o agencia de los propios movimientos, y se de-construyen las unidades de acciones con sentido». La persona ya no está en condiciones de llamar suyos a muchos de sus movimientos, los considera extraños, diferentes de sí. Ya no se median silenciosa o implícitamente con el mundo, sino que requieren atención explícita, deliberación para su ejecución y control de cada uno de los movimientos necesarios y presentes en un esquema. En estas condiciones, cabe hablar propiamente de una disolución de la intencionalidad corporal. El paciente entonces vive una escisión entre su conciencia y su corporalidad, que puede llevar a anular casi cualquier acción corporal mínimamente compleja del agente.

\section{III.2. La pérdida de la inmediatez medida}


Como dijimos, Thomas Fuchs suele citar la caracterización que hace Helmuth Plessner de nuestro estar inmediatamente en el mundo circundante, como una inmediatez mediada - vermittelten Unmittelbarkeit- corporalmente. El conjunto de mediaciones corporales que nos permiten percibir y actuar en el mundo, generalmente son silenciosas, tácitas. Sólo somos conscientes de ellas en los procesos de aprendizaje, después nuestra conciencia las da por descontado para poder dedicarse a otras actividades que solemos denominar por ello superiores. Así estas mediaciones corporales constituyen el conjunto de hábitos más o menos sistemático o coherente que nos familiarizan con las diferentes dimensiones y elementos de nuestro cuerpo, con nuestras actividades cotidianas de habitación del mundo, y con las otras subjetividades con quienes interactuamos.

Pues bien, esta mediación tácita de nuestro cuerpo puede verse alterada. Entonces nuestros hábitos aparecen como extraños. La conciencia vuelve sobre las técnicas corporales adquiridas como observándolas con extrañeza. En lugar de vivir inmediatamente gracias a nuestros hábitos, observamos esos hábitos, los objetivamos, y por eso nuestro cuerpo, sus elementos, y sus esquemas de acción, devienen extraños — alieni-objetos que hay que mover desde fuera, privados de un movimiento per se, y por ello mecánicos.

Para S.N. y L.N., por ejemplo, ver se ha transformado en mirar a las imágenes a través de una cámara. Ellos perciben el percibir. En tanto que estas experiencias no son vividas desde dentro, pueden ser observadas como objetos o datos reificados. Podríamos denominar a esto una mecanización del experienciar (Haan, Fuchs, 2010: 331).

Los diferentes elementos de la corporeidad se transforman en objetos, el cuerpo en una máquina complejísima, los diferentes esquemas corporales en sus mecanismos subordinados que no se logran dirigir, dominar, ni reproducir correctamente desde la conciencia. El cuerpo ya no es cuerpo vivido y viviente — Leib- Es justamente cuerpo objeto y objetivado — Körper-. Siente su cuerpo fragmentado en diferentes partes. El sujeto vive la disolución de su Leib, de sus hábitos o técnicas corporales. La conciencia no se comunica con ellos, se ha desconectado de ellos: 
La desconexión entre el cuerpo y la mente altera fundamentalmente a las dos: la mente deviene un observador hiperreflexivo, el cuerpo una máquina hiperautomática. Los dos están "desanimados", como dijo Stanghellini (Haan, Fuchs, 2010: 331).

\section{III.3. Las vivencias esquizofrénicas de control ajeno del cuerpo}

La pérdida de inhabitación del propio cuerpo es bastante patente en vivencias muy características de la esquizofrenia en las que los pacientes aducen un control ajeno de su corporeidad. Alguien extraño, por ejemplo, es el que mueve sus brazos o sus piernas. ¿Cómo explicar esta vivencia tan característica del esquizofrénico? La hipótesis habitual es cognitiva: la sensación de control ajeno del propio cuerpo sería un delirio, un trastorno cognitivo, en modo alguno relacionado con las interacciones del sujeto con otros. La de Thomas Fuchs en cambio se basa en la tesis merleau-pontiana sobre la transferencia de los esquemas corporales, explorando cómo se realiza esta transferencia en personas cuya corporalidad propia es vivida como irreal.

La hipótesis cognitiva sostiene que la sensación de control ajeno del propio cuerpo se debe a un delirio que lleva al esquizofrénico a atribuir a un agente extraño la causa de los esquemas corporales que actualmente adopta. Pero ¿y si el cuerpo propio es percibido no como propio, si la irrealidad que orla las vivencias del esquizofrénico se extendiera también al propio cuerpo? Es el propio esquema corporal el que el paciente esquizofrénico percibe como extraño, como ajeno. Entonces, el esquizofrénico tiene que hipotetizar sobre los propios movimientos de su propio cuerpo. ¿Por qué se mueve como se mueve? Como dijimos, este como es el conocimiento implícito, directo e inmediato que denominamos esquema corporal. Según Fuchs, en el esquizofrénico el esquema corporal es lo que se halla debilitado. Por eso, porque lo siente como ajeno, es por lo que necesariamente, es decir, como cualquier sujeto sano, transfiere los esquemas corporales que aún puede reconocer como propios al movimiento que no percibe como propio:

[...] podemos hipotetizar que en la esquizofrenia, con el debilitamiento del sentido de la agencia, acaece un cortocircuito del modelado de la acción, y se pierde la discriminación crucial entre la acción de uno y la del otro. Así el "como sí" virtual 
expuesto por Merleau-Ponty deviene un "como sí" actual para el paciente: "Es como si otra persona estuviese tras mi moviendo mis brazos". Esto conduce finalmente a la falsa atribución delirante de los movimientos a un agente extraño. Los delirios de control no se basan por tanto en inferencias cognitivas equivocadas, sino en un sentido inmediato de alteridad en el mismo movimiento vivido (Fuchs, 2005: 103).

Así, la sensación de control del propio cuerpo tiene como causa - ésta es la hipótesis de Fuchs-, la incapacidad de establecer correctamente la distinción yo-otro, es decir, responde a un menoscabo en la habilidad intersubjetiva básica yo respecto del primer polo de la estructura yo-otro. La sensación de que mi cuerpo es controlado por otro es una falla en mi autoconciencia corporal, una descorporalización de mi propio ego. Una instalación del yo fuera de su sí mismo somático. Pero lo es, no porque como un filósofo busque reflexivamente motivos de duda respecto de la existencia de su cuerpo, o porque quiera examinar el mundo en que vivía, los lazos intersubjetivos que tenía tras un desengaño o una crisis existencial. La instalación del yo en la reflexión en el caso del paciente esquizofrénico tiene como fundamento la disminución de su capacidad de actuar en el mundo. Aparece una merma en sus intereses, y en las respuestas que su cuerpo ofrece a las solicitaciones que las cosas le dirigen. Por este déficit de su intencionalidad corpórea atribuye sus propios movimientos a un agente externo.

\section{III.4. Hiperreflexividad}

El sujeto, puesto que el sí mismo vital o yo básico se ha disuelto se confina, cartesianamente, en su conciencia reflexiva. Existe meramente en el polo del yo extendido o reflexivo. Con frecuencia esta instalación en la reflexión es aterradora y angustiosa, pues el paciente se observa obsesivamente desde su yo reflexivo. Es decir, exacerba su reflexión, se comporta hiperreflexivamente. Observa ese yo básico que está en trance de disolución, aquello que ya no puede llamar sus sensaciones, movimientos, acciones, sus esquemas corporales. «Eso» ya no es como era, resulta extraño — alienum -; lo antaño más familiar hogaño resulta lejano. De Haan y Fuchs lo ejemplifican con el siguiente testimonio clínico: 
Constantemente tenía que preguntarme quién era yo realmente. Es difícil explicarlo .... La mayor parte del tiempo me encontraba ante esta cosa extraña: me veo de cerca, cómo soy, qué hago ahora y donde están las "partes" ... Pienso acerca de esto tanto que no puedo hacer ninguna otra cosa. No es fácil cuando cambias día a día. Como si fueras una persona totalmente diferente de repente (Haan, Fuchs, 2010: 329).

Instalada la persona ahí, en sus vivencias puramente subjetivas se desconecta por una parte del mundo que la rodea y en el que habita, y también de su propio cuerpo, que ahora en sus movimientos y gestualidad, no la manifiesta. Además, la mayoría de las acciones requieren una atención especial consciente (hiperreflexividad), o se vive el propio cuerpo como si fuera un robot o una máquina (hiperautomatismos).

Además, instalada en su conciencia reflexiva, y desconectada de su corporalidad, el fenómeno de la resonancia corporal queda en entredicho. Su mímica corporal, su expresividad, y sus preferencias lingüísticas no son el responder inmediato y directo al mundo que la circunda o a los otros:

[...] las expresiones emocionales y verbales no parecen corresponder con el otro o con el contexto; los movimientos corporales y expresiones no están integradas para formar un todo armonioso a través del cual la persona podría manifestarse (Fuchs, 2015: 201).

Quien interactúa con el autista o el esquizofrénico no encuentra, en determinadas ocasiones de crisis, resonancia en su mímica corporal ni en su expresividad ni en su gestualidad. Éste parece estar ausente del mundo en que habitan los otros, encerrado en el mundo que suscitan las alucinaciones y voces que siente. La conexión con el mundo común está rota.

Por eso, el paciente esquizofrénico siente que las relaciones con otros se vuelven cada vez más difíciles y complicadas La ausencia de resonancia corporal en el paciente autista o esquizofrénico, según Fuchs, es indicativa de su aislamiento, de su incomunicación. Mis intenciones no inhabitan su cuerpo. Por eso, mi cuerpo, en lugar de ser percibido por él como el cuerpo de una subjetividad viviente, es en cambio percibido como un cuerpo-objeto -Körper -, no como mi cuerpo viviente y vivido — Leib-. 


\section{El problema de los delirios y alucinaciones}

La hipótesis de Fuchs sobre la esquizofrenia, es decir, considerarla a ésta como un trastorno de la intersubjetividad encarnada y no principalmente como un trastorno neurológico o cognitivo, ha de enfrentarse al problema de los delirios.

[...] los delirios son fenómenos relacionales, precisamente porque escapan a nuestros intentos de comprensión; se manifiestan a través de la negación del orden establecido de los sentidos por el cual pretendemos comprenderlos (Fuchs, 2015: 208).

Los delirios son alteraciones perceptivas y cognitivas en general. Además, está el hecho de que la esquizofrenia afecta principalmente, como hemos visto, a la autoconciencia del sujeto. Bien es verdad que hemos examinado que el trastorno reside en la autoconciencia vital o yo básico, y no a la autoconciencia reflexiva o el yo tal y como comparece ante el intelecto. La presencia de delirios y la afectación de la autoconciencia parecen avalar la tesis cognitivista sobre la esquizofrenia. La tesis neurológica parece avalada por el hecho de que muchos delirios y alucinaciones concurren en enfermedades somáticas y no mentales, por lo que en última instancia podría encontrarse la causa neural de las mismas.

La hipótesis de Fuchs, como hemos mostrado, difiere de uno y otro enfoque:

En lugar de cosificar el delirio y situarlo como un estado localizable en la cabeza del paciente, un enfoque enactivo lo considera como una perturbación de la intersubjetividad, que surge en una situación social que siempre está constituida por dos o más interlocutores (Fuchs, 2015: 204).

Recordar la diferencia entre delirio y alucinación nos será de ayuda para comprender este texto de Fuchs. La alucinación es una ficción por completo mental. La mente urde o trama situaciones, percepciones multimodales, etc. por completo irreales. En este sentido las alucinaciones son entidades de razón, autónomas, independientes de lo que hay, en uno o en el mundo. En 
cambio, el delirio no se deja cosificar tan fácilmente. El delirio no es independiente de los movimientos o del cuerpo con los que se ha perdido contacto vital y que se perciben como irreales. El delirio es propio de la instalación hiperreflexiva en la conciencia intelectual observando u objetivando las propias percepciones, movimientos y pensamientos. Como objetivación es una toma de distancia respecto de la actividad psíquica y vital. Un poner frente al propio pensamiento aquello con lo que inmediatamente nos instalamos en el mundo. Entonces aparece la extrañeza frente a esas actividades. Al tomar el protagonismo no se realizan de manera tácita. Y el paciente, extrañado, alienado, siente enigmáticas las operaciones que principia su naturaleza. En estas condiciones, al ver extrañas sus operaciones, las des-naturaliza, las considera como artificiales, mecánicas, etc. Así, por ejemplo, identifica su percibir con la actividad de una cámara cinematográfica, los movimientos de su cuerpo, como dirigidos o controlados desde fuera, y sus pensamientos como insertados o producidos por un chip.

\section{Conclusiones}

En este artículo se ha tematizado la distancia que hay entre la vida y la reflexión, entre la conciencia vital y la reflexiva, entre el yo básico y el yo extendido. La investigación que ahora concluye arrancó de motivos muy vitales, en los que estaba muy implicado mi yo básico: mi padre padecía esquizofrenia. La reflexión filosófica arrancó de mis propias vivencias, de sus sufrimientos y los de mi familia. Este trabajo, creo, ha sido un esfuerzo de comprensión, de saber qué vivía mi padre y cómo lo vivía. Sabía en efecto por qué. La causa estaba identificada, tenía un nombre. Los síntomas estaban claros, el tratamiento dando sus resultados, y el pronóstico, también bastante bien perfilado. Pero todas las explicaciones científicas que habíamos recibido en esos años, no nos orientaban lo más mínimo en la comprensión de qué vivía y de cómo lo vivía. Por eso, ese esfuerzo de comprensión que he realizado en estos meses desde que oí hablar de Thomas Fuchs y de la fenomenología de la psiquiatría a Juan José Padial, son también un esfuerzo de orientación, o como diría Jaspers, de iluminación existencial filosófica — philosophisches 
Existenzerhellung-, de saber dónde y cómo se encuentra mi padre en su existencia.

Por eso, la conclusión a este artículo debe volver sobre las vivencias en las que tuvo su comienzo. Sólo así podré ajustar cuentas entre mi reflexión y la vida que intenta comprehender o conceptualizar. Sólo así podré ver si mis estudios en psiquiatría fenomenológica han girado en el vacío, o realmente iluminan nuestra existencia.

He explorado despaciosamente la hipótesis de Thomas Fuchs sobre la esquizofrenia. Ésta no es primariamente un trastorno cognitivo, ni meramente neurológico. Es un trastorno de la subjetividad encarnada, y por ello un trastorno de la persona, que asiste extrañada — alienada - al disolverse de algunas dimensiones constitutivas suyas. Ya no puede llamar suyos a sus esquemas corporales, a sus sentimientos, percepciones y pensamientos. $\mathrm{Y}$ a veces, ni siquiera a su cuerpo. Una anécdota, creo que sumamente hermosa, vivida con mi padre, ilumina que esta disolución del esquema corporal, de la pérdida de una parte de su tener y su poder, no era total. La encarnación es algo profundo, misterioso. $\mathrm{Y}$ aunque disuelta, las relaciones de la persona con su cuerpo, son estrechas, hasta el punto de que esa disolución es más un letargo que una pérdida: una tarde de paseo con mi padre, mis hijos iban en bicicleta. Mi padre creyó que mi hijo se iba a caer y salió corriendo con sorprendente agilidad y vitalidad. Normalmente arrastraba los pies muy lentamente. Mi hijo no se cayó, y mi padre que había corrido en su auxilio, súbitamente dejó de hacerlo, y volvió al andar inseguro y torpe, vacilante, con el que intentaba controlar hiperreflexivamente ese cuerpo del que se ha distanciado.

Esta investigación ha expuesto los esfuerzos de Fuchs por proseguir el tema de la intercorporalidad -intercorporéité- esbozado por MerleauPonty. Este filósofo sólo pudo proponerlo. Me parece que Fuchs lo desarrolla y además muy acertadamente. La anécdota anterior ilustra muy bien el entrelazamiento prerreflexivo entre su cuerpo y el cuerpo viviente de mi hijo. Demuestra como su cuerpo viviente, el cuerpo de una persona - un cuerpo de carne y hueso- era afectado por los movimientos del cuerpo de mi hijo. Es una anécdota de la comunicación encarnada entre dos sujetos encarnados o personas humanas.

Que la comunicación —y la intersubjetividad — es primariamente encarnada lo muestra otra vivencia con mi padre. Si la primera fue puntual, esta era habitual. A mi padre siempre le había gustado jugar al dominó con sus amigos. 
Por eso nosotros buscábamos jugar con él. Lo hacíamos, aunque nos gane en muchas ocasiones. Contaba las fichas y empleaba jugadas bien razonadas. Sin embargo, era incapaz de seguir el turno. Siempre creía que le tocaba mover ficha a él. Incluso aunque lo acabara de hacer. La dificultad de este juego estriba en el cálculo de las fichas que hay sobre la mesa y las que quedan por ponerse. No es un juego manual, sino intelectual. Su cuerpo no era atraído, ni solicitado, por otro cuerpo, como el de mi hijo en peligro. Sólo es atraída su mente. No se establece ningún diálogo entre dos corporeidades, sino un monólogo de la mente consigo misma en torno a la posibilidad de poner una ficha. La disolución de sus esquemas corporales, y la hiperreflexividad, aíslaban a mi padre. Hacían prácticamente imposible — desaparecidas la resonancia corporal - las relaciones intersubjetivas, puesto que el lugar natural y primario de estas es la intercorporalidad y la interafectividad.

Quizá por ello percibía tanta distancia en la comunicación inmediata y directa con mi padre. No encuentro en él resonancia corporal de mis intenciones, afectos, emociones, etc. Su cuerpo, como lo vivo yo, no entablaba ese diálogo prerreflexivo con el mío. No sólo su cuerpo se había objetivado - Körper y no Leib-. También mi cuerpo era objetivado por él. Y quizá también por ello, no era posible entablar una verdadera comunicación, una apertura al otro. Sea cual sea el tema de conversación que trataras de entablar, él siempre iba a tratar lo mismo, te contaba las cosas que las voces le han dicho o los mensajes que le mandaban a través de la televisión. Te contaba, en suma, lo que aparecía en su conciencia reflexiva, en su yo extendido.

Y por ello, por esa instalación hiperreflexiva en su conciencia, deliraba. Escuchaba su propio escuchar. Y eso, es lo que nos decía, desencajada la cara, aterrorizado. Alguna vez me había preguntado si yo no estaba escuchando o viendo lo que él veía. Su mirada estaba extrañada, su cuerpo en tensión. A distancia de mí, y de lo que veía y oía. Extrañado, alienado.

Esta conclusión puede parecer poco científica y poco apropiada para culminar un artículo en una prestigiosa y especializada revista en filosofía. Pero es una reflexión sobre la vida, una reditio in seipsa reditione completa, una vuelta del cognoscente sobre sí mismo con una vuelta completa, que es como Proclo definió la reflexión, como Tomás de Aquino dijo que el alma se conoce a sí misma esencialmente, y como Hegel definió la filosofía. 


\section{Bibliografía}

A) De Thomas Fuchs.

Froese, T., Fuchs, Th. (2012): «The extended body: A case study in the neurophenomenology of social interaction», Phenomenology and the Cognitive Sciences (11/2012: 205-236).

Fuchs, Th. (2001): «The tacit dimension» Philosophy, Psychiatry, \& Psychology, (8/2001: 323-326)

Fuchs, Th. (2005): «Corporealized and Disembodied Minds. A Phenomenological View of the Body in Melancholia and Schizophrenia», Philosophy, Psychiatry, \& Psychology, 12/2.

Fuchs, Th. (2009), De Jaegher.: «Enactive intersubjectivity: Participatory sense-making and mutual incorporation», Phenomenology and the Cognitive Sciences (8/2009: 465-486).

Fuchs, Th., (en línea): «Are Mental Illnesses Diseases of the Brain?» DOI: 10.1002/9781444343359.ch16. (2011: 331-344). https:/www.researchgate.net/publication/229975325_Are_Mental_Illnesses_Diseases_of_the_Brain [Consultado: 20/08/2020]

Fuchs, Th. (2014: 75): «Brain Mythologies. Jaspers' Critique of Reductionism from a Current Perspective», T. Fuchs et al. (eds.), Karl Jaspers' Philosophy and Psychopathology, Springer, Nueva York.

Fuchs, Th. (2016): «Intercorporality and interaffectivity», Phenomenology and Mind, (11/2016: 200).

Fuchs, T (2017:83-98): «The As If function and its loss in Schizophrenia», DOI: 10.4324/9781315411538-5.

Fuchs, Th., Röhricht, Fr. (en línea): «Schizophrenia and intersubjectivity. An embodied and enactive approach to psychopathology and psychotherapy» en Philosophy, Psychiatry \& Psychology, Junio (2017), https://www.researchgate.net/publication/309902549

[Consultado: 20/08/2020]

Fuchs, Th. (2020): «Delusion, Reality, and Intersubjectivity: A Phenomenological and Enactive Analysis», Philosophy \& Psychiatry. Johns Hopkins University Press Volume 27, Number 1, (2020: 61-79), DOI:10.1353/ppp.2020.0009.

Fuchs, T. (en línea): «Pathologies of Intersubjectivity in Autism and Schizophrenia», Journal of Consciousness Studies, (2004) $\mathrm{n}^{\circ}$ 22, pp. 191-214 https://www.uniheidelberg.de/md/philsem/phaenomenologie/pathologies_of_intersubjectivity.pdf

[Consultado: 19/08/2020]

Haan, S., Fuchs, Th. (2010): «The Ghost in the Machine: Disembodiment, SchizophreniaTwo case Studies» Psychopathology 2010;43:327-333 DOI: 10.1159/000319402.

B) De otros autores.

Gallagher, S. (2001): «The practice of mind: theory, simulation or primary interaction? », Journal of Consciousness Studies (8/2001: 83-108).

Gallagher S. (2004): «Neurocognitive models of schizophrenia: a neurophenomenological critique», Psychopathology; (2004,37: 8-19).

Henry, M. (2000): Incarnation. Une philosophie de la chair. París: Seuil.

Jaegher, H., Di Paolo, E. (2007): «Participatory sense-making: An enactive approach to social cognition», Phenomenology and the Cognitive Sciences, (6/2007:485-507). 
Merleau-Ponty, M. (1960): Le philosophe et son ombre. Signes. París: Éditions Gallimard.

Merleau-Ponty, M. (1945): «Phénomenologie de la perception», Gallimard, París. Citado por Fuchs, Th., «Corporealized and Disembodied Minds. A Phenomenological View of the Body in Melancholia and Schizophrenia», PPP, 12/2, (2005:98).

Pedrosa, Sara. (2018): Comunicación en intercorporalidad en la fenomenología de la psiquiatría Thomas Fuchs. Trabajo de fin de máster, Universidad de Málaga.

Sartre, J.-P. (1943): L’Etre et le néant. París: Gallimard. capítulo I de la segunda parte.

Sass L. A., Parnas J. (2003), «Schizophrenia, consciousness, and the self», Schizophrenia Bull; 29: 427-444.

Zahavi, D., (2005): «Subjectivity and selfhood: Investigating the first-person perspective», MIT Press, Cambridge, MA.

\section{Rosa María de Barrio Rodríguez rmdebarrio@gmail.com}

